\title{
Using CPTs to derive thermal properties of soil
}

\author{
Philip J. Vardon ${ }^{1,{ }^{*}}$ and Joek Peuchen ${ }^{2}$ \\ ${ }^{1}$ Delft University of Technology, Section of Geo-Engineering, PO Box 5048, 2600GA Delft, The Netherlands \\ ${ }^{2}$ Fugro, PO Box 130, 2630 AC Nootdorp, The Netherlands
}

\begin{abstract}
A method of utilizing cone penetration tests (CPTs) is presented which gives continuous profiles of both the in situ thermal conductivity and volumetric heat capacity, along with the in situ temperature, for the upper tens of meters of the ground. Correlations from standard CPT results (cone resistance, sleeve friction and pore pressure) are utilized for both thermal conductivity and volumetric heat capacity for saturated soil. These, in conjunction with point-wise thermal conductivity and in situ temperature results using a Thermal CPT (T-CPT), allow accurate continuous profiles to be derived. The CPT-based method is shown via a field investigation supported by laboratory tests to give accurate and robust results.
\end{abstract}

\section{Introduction}

Measuring or estimating the thermal properties of the ground in situ in the upper 10s of meters is essential for safe and economical design of various energy geostructures, cables and pipelines.

Obtaining accurate in situ thermal properties is problematic, as laboratory methods involve sampling material, which can easily change density or saturation and in situ methods often give bulk parameters, involve long tests and/or equipment which is susceptible to break. It has been observed that laboratory and in situ testing give different results $[1,2]$. Most common methods only determine the thermal conductivity and are unable to determine the volumetric heat capacity of the soil.

This paper presents a robust approach for obtaining accurate in situ continuous profiles for both the thermal conductivity and the volumetric heat capacity for saturated soil. This approach builds upon the strengths of two recently proposed in situ methods.

\section{Methods}

\subsection{Laboratory methods}

\subsubsection{Needle probes}

Laboratory needle probes [3] operate by applying a constant heat flux (via the application of a constant electrical power) evenly to a thin needle and the temperature rise in the needle is measured. The heat is assumed to conduct radially through the soil material, and a line source analytical solution [4] is utilized to estimate the thermal conductivity. Both the heating and cooling phases can be used to derive the thermal conductivity. The needle must be thin enough so that the analytical solution is still reasonable (e.g. ASTM D5334-14 [3]). The (volumetric) heat capacity cannot be determined.

The advantages of the method are that it is relatively easy to carry out and is reasonably quick. However, to gain data representative of in situ conditions, densities, stress states and saturation levels must be known and maintained.

\subsubsection{Transient plane}

The transient plane test [5], often referred to as a Hot Disc test, works in a similar principle as the needle probe, but with a flat (plane) heating element which is typically placed between two samples of the same material. An analytical solution is utilized to calculate both the thermal conductivity and the volumetric heat capacity. Therefore, the heat diffusivity can also be calculated [5].

The method is fast, typically requires smaller samples than needle probe tests and derives both thermal conductivity and volumetric heat capacity but requires a certain skill level to carry out. As with other laboratory tests, for the results to be representative of in situ conditions, these conditions must be known and maintained during the test.

\subsection{In situ methods}

\subsubsection{Thermal response tests}

The thermal response test $[6,7]$, known commonly as a TRT, involves the installation of a U-tube heat exchanger in the ground. Typically, a constant power is then applied by circulating a fluid in the U-tube with a constant temperature difference between the two legs. If carried out in this way, the interpretation is similar to

\footnotetext{
* Corresponding author: p.j.vardon@tudelft.nl
} 
that of a needle probe. Conversely, other thermal regimes (e.g. constant input temperature) may be used and distributed measurements can be used along the borehole. In those cases, alternative analytical or numerical tools can be used to interpret the test (see, for example, [8] for more details).

Thermal response tests are often carried out on a site where several ground source heat pump heating systems will be installed. A TRT can then be carried out in one of the boreholes. This has the advantage of testing the combination of layers that will be used for these systems and can measure substantial depths (10s to $100 \mathrm{~s}$ of meters). The tests typically last 2 days, and give a bulk thermal conductivity for the tested soil [8]. The longtime taken makes the test vulnerable to the influence of ground water flow (convection) as well as conduction.

\subsubsection{In situ needle probe}

A range of needle probes are available for in situ use. These operate exactly as the laboratory test, however either require a pre-drilled hole, which may require casing, or require the needle to be pushed or vibrated to the correct depth. In the first of these cases, a substantial expense is likely to be incurred and for the second, the tool can have inadequate penetration or is vulnerable to break. The test itself is fast to perform (albeit probably longer than in the laboratory due to the required increase in dimensions for strength of the needle part of the probe).

Various variants have been proposed each with specific characteristics that determine the range of conditions under which operation is possible.

\subsubsection{Thermal cone (T-CPT)}

The thermal cone penetration test (T-CPT) or thermal cone test was proposed by [9] and a thorough interpretation method was proposed by [1], based on an analytical solution [10]. CPTs [11] are used to estimate soil properties by measuring the response of a cone penetrometer continuously pushed though the soil. The test takes advantage of heat which is typically generated from friction during the normal operation of a cone penetration test (CPT), which results in the penetrometer heating up. The test is interrupted, i.e. paused, and the heat is allowed to dissipate. The temperature dissipation over time is used to interpret the thermal conductivity.

The method [1] utilizes two steps to get to the thermal conductivity, the first step is to calculate the in situ temperature, using [1]:

$$
T_{0}=\frac{t_{1} T_{1}-t_{2} T_{2}}{t_{1}-t_{2}}
$$

where $T_{0}$ is the in situ temperature, $T$ is the measured temperature at time $t$, where the subscripts 1 and 2 relate to an earlier and later time, respectively. After some time (i.e. a few hundred seconds, depending on the cone diameter), the value converges and that is the estimated in situ temperature used in the second step. It is not necessary for the measured temperature to reach the in situ temperature.

The second step is the determination of thermal conductivity, using [1]:

$$
k=f_{T C} \frac{S\left(T_{\max }-T_{0}\right)}{4 \pi\left(t\left(T-T_{0}\right)\right)}
$$

where $k$ is the thermal conductivity, $f_{T C}$ is a cone calibration factor (which is cone specific and is calculated numerically), $S=c_{p} A \rho$ is the heat content per unit length per degree (where $c_{p}$ is the specific heat capacity, $A$ is the cross sectional area and $\rho$ is the density of the cone penetrometer material) and $T_{\max }$ is the maximum temperature recorded. An equivalent graphical method can also be used, see [1] for details. The equation is based upon the heat content of the cone penetrometer dissipating over time and the theory presented by [10].

The method is passive, so does not require a heater, but does require the addition of a temperature sensor in the cone. It is fast; a single test takes about 300s. Several measurements can be taken in a vertical profile, and typical CPT measurements can be taken at the same time (a major advantage for energy geo-structures). A measurement of in situ temperature is also made at the same time. It is limited to the depths that CPTs can operate over (several $10 \mathrm{~s}$ of meters), and requires friction to be sufficient to heat up the cone penetrometer. This implies that soil profiles with only normally consolidated or lightly over-consolidated clays or organic material cannot be easily tested. However, those materials can be tested if sufficient heat is generated by first going through layers with sufficient friction. The method has been seen to be accurate in comparison to laboratory and field tests, as long as the cone temperature is approximately $>3^{\circ} \mathrm{C}$ above the in situ temperature [1]. Temperatures generated in different soils are presented in [1] and [9].

Theoretically, the volumetric heat capacity could also be captured, but the data are contained within the first few seconds of temperature dissipation and as a consequence it has little precision [1].

\subsubsection{CPT correlations}

The thermal properties of a soil vary mainly due to the composition and density of the soil, as well as the saturation level in unsaturated soils. As CPTs are regularly used to estimate the soil behavior type, which correlates closely with the composition and the saturated density, the thermal properties can also be estimated by use of correlations with the standard CPT measurements, i.e. cone resistance, sleeve friction and pore pressure [12]. Two of the methods of [12] are summarized here. The methods are based on density correlations for saturated soil and expected thermal properties of soil components. The methods have been tested against experimental data in [12].

Figure 1 presents a correlation for thermal conductivity, which makes use of a Soil Behavior Type (SBT) chart, particularly normalized cone resistance, $Q_{t n}$, 
and normalized friction ratio, $F_{r}$. At high cone resistances this correlation over-estimates the thermal conductivity and requires a correction. Figure 2 provides a correlation for the volumetric heat capacity, $C$, proposed by [12].

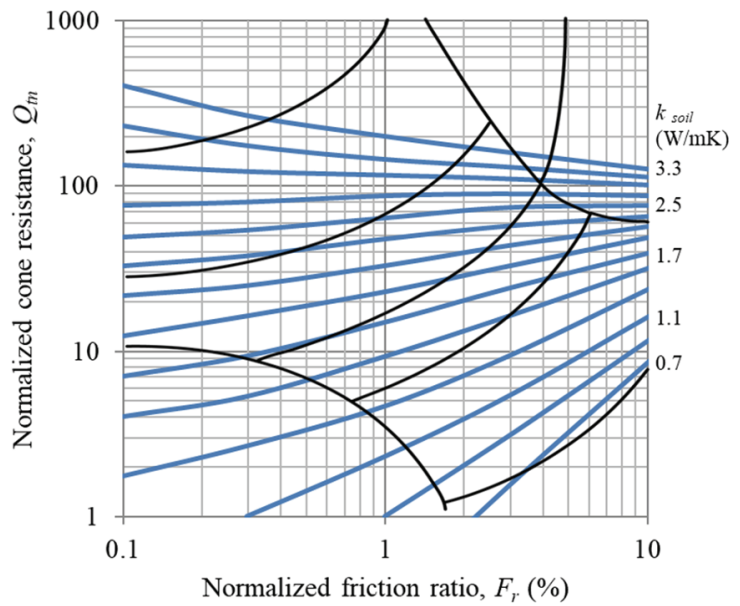

Fig. 1. Thermal conductivity correlation.

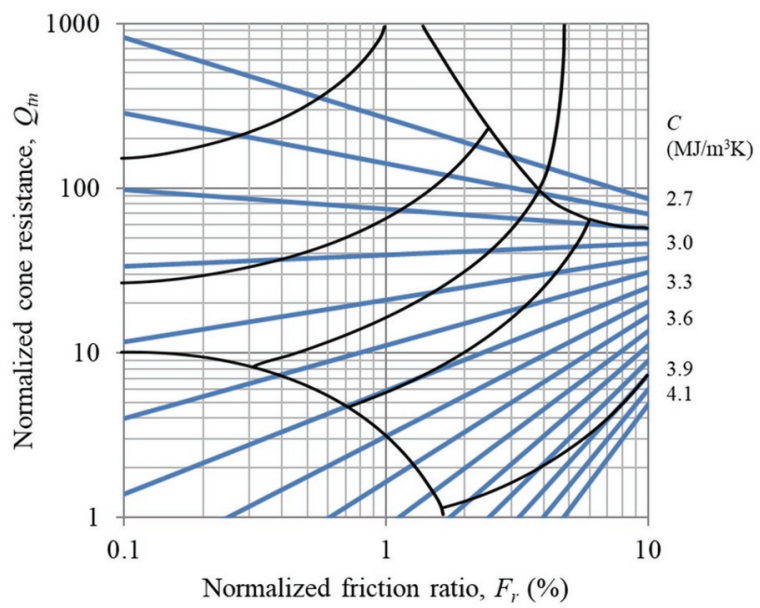

Fig. 2. Volumetric heat capacity correlation.

These correlations offer real-time determination of complete profiles of thermal properties (both thermal conductivity and volumetric heat capacity), as part of conventional CPT data acquisition. It has been seen that the correlation for volumetric heat capacity is relatively accurate and precise (i.e. robust) whereas the thermal conductivity is accurate but less precise (i.e. has a larger error) and deviates from the true values for dense sands [12]. For information on the accuracy and comparison with different types of collected data (field and laboratory), the reader is referred to [12].

\subsection{Combined CPT method}

This paper illustrates a method combining the advantages of both the thermal cone and the CPT correlations (presented in Figure 3). This method is able to provide continuous profiles of thermal properties in the upper 10s of meters of ground. At the same time, a standard CPT investigation is carried out, leading to an efficient and effective site investigation for energy geostructures.

Depending on the amount of CPTs required for the site investigation, the strategy can differ. If many CPTs are required, first an initial continuous CPT with a temperature measurement can be carried out. Layering of the soil is identified to guide the locations of later TCPTs and temperatures are monitored to determine whether sufficient heat is contained in the cone penetrometer to achieve a successful T-CPT. Volumetric heat capacity profiles are determined and thermal conductivity profiles initially estimated. Secondly, select $\mathrm{T}-\mathrm{CPTs}$ are carried out targeting (i) center of select layers, (ii) locations with high normalized cone resistance (high is defined as above approximately 170).

If only few CPTs are required, firstly a T-CPT can be carried out with temperature dissipation tests carried out regularly, for example every $1.5 \mathrm{~m}$ or when the temperature rise is sufficient, i.e. $>3^{\circ} \mathrm{C}$. Secondly, further T-CPTs are carried to target any missing layers.

In both strategies, the thermal conductivity profiles based on correlations are corrected by using the pointwise information. As with all methods based on correlations, site-specific or area-specific correlations can improve estimations. The corrections can be carried out separately for each soil layer. Where considerable deviation is observed, or a large site investigation is undertaken, additional T-CPTs at multiple locations in each layer would yield more reliable information.

Two corrections can be carried out, the first for high normalized cone resistance being:

$$
k_{\text {corr }}=k_{\text {orig }}-a \log \left(Q_{t n} / Q_{t n \_ \text {lim }}\right) \text { if } Q_{t n}>Q_{t n \_l i m}
$$

where $k_{\text {corr }}$ is the corrected thermal conductivity, $k_{\text {orig }}$ is the thermal conductivity estimated from the CPT correlations, and $Q_{t_{-} \text {lim }}$ is a value above which the thermal conductivity is over-estimated. $a$ is a parameter controlling the magnitude of the correction.

The second correction is proposed to address a systematic over or under estimation. It is only based on normalized cone resistance, as this has the greatest impact on the results from the thermal conductivity correlation (as can be seen in Figure 1), and having only one parameter makes it more straightforward to estimate. The correction is proposed to be:

$$
k_{\text {corr }}=k_{\text {orig }}-b-c \log \left(Q_{t n} / Q_{t n_{-} \text {mean }}\right)
$$

where $k_{\text {corr }}$ is the corrected thermal conductivity, $k_{\text {orig }}$ is the thermal conductivity estimated from the CPT correlation (possibly already corrected by Eq. 3) and $Q_{t n \text { mean }}$ is the layer mean normalized cone resistance. The parameters $b$ and $c$ control the offset and range of thermal conductivity correction, respectively. Particular soil characteristics, such as soil structure, can result in an offset. A default of $b=0$ and $c=0$ is considered standard, without further information. 


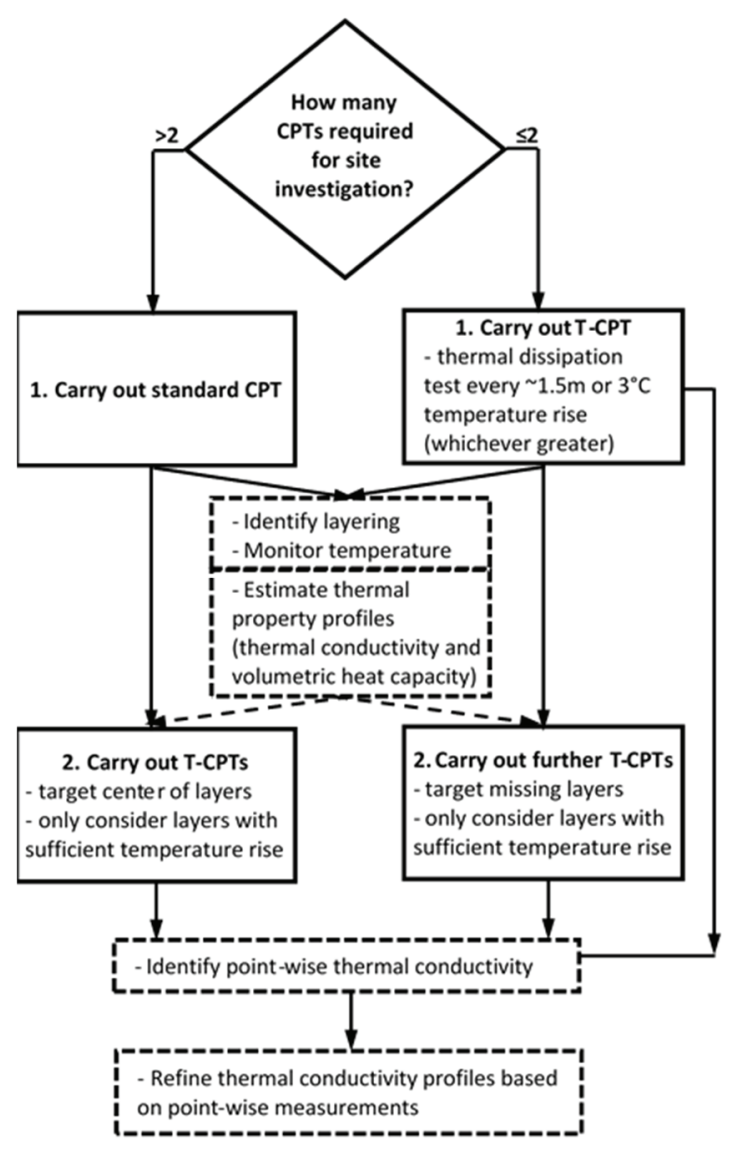

Fig. 3. Example flowchart of the proposed method for CPTbased thermal properties determination. Solid boxes represent test steps, dotted boxes represent data processing steps, solid arrows represent order of steps and dotted arrows represent a data flow.

\section{Case Studies}

Data have been obtained from a number of site investigations where a range of CPTs, T-CPTs, in situ needle probes and laboratory needle probe tests are available.

The sites, with the available data, are:

1. Ostend site (Belgium): CPT, laboratory needle probe. This site was investigated for the installation of energy piles and comprised 4 CPTs and 8 laboratory needle probe tests on undisturbed samples taken at various depths.

2. Delft site (Netherlands): CPT, T-CPTs, in situ needle probe, laboratory needle probe. This site is the location of an experimental energy pile, and was used as part of the validation of the T-CPT. There are 4 CPTs, $10 \mathrm{~T}$-CPT points (taken during the CPTs), 2 in situ needle probe tests and 13 laboratory needle probe tests on undisturbed samples taken at various depths.

For both sites the water table was around $1 \mathrm{~m}$ below the surface. The results from the process given in Section 2.3 are outlined in Figures 4 and 5. The two figures show the basic CPT data on the left. Note that the CPT data for the Ostend site and Delft site are for $0.2 \mathrm{~m}$ and $0.02 \mathrm{~m}$ depth spacing, respectively. Profiles of volumetric heat capacity and (uncorrected and corrected) thermal conductivity are shown on the right, with an identification of layers at the depths of point measurements. The thermal conductivity profiles include the discrete (point) thermal conductivity data. The correction parameters for each layer (counting from the surface) are given in Table 1 . The thermal conductivity data match the uncorrected profiles reasonably or well depending on the layer, and with relatively small adjustments match the trends and values accurately in the corrected profiles.

Table 1. Correction parameters per layer.

\begin{tabular}{|c|c|c|c|c|c|}
\hline Site & Layer & $\boldsymbol{a}$ & $\boldsymbol{b}$ & $\boldsymbol{c}$ & $\boldsymbol{Q}_{\boldsymbol{t n} \_ \text {lim }}$ \\
\hline \multirow{5}{*}{ Ostend } & 1 & 1.7 & 0 & 0 & 170 \\
\cline { 2 - 6 } & 2 & 1.7 & -0.5 & 0.5 & 170 \\
\cline { 2 - 6 } & 3 & 1.7 & 0 & 0 & 170 \\
\cline { 2 - 6 } & 4 & 1.7 & -0.4 & 0.3 & 170 \\
\cline { 2 - 6 } & 5 & 1.7 & 0 & 0 & 170 \\
\hline \multirow{5}{*}{ Delft } & 1 & 1.7 & 0 & 0 & 170 \\
\cline { 2 - 6 } & 2 & 1.7 & 0 & 0 & 170 \\
\cline { 2 - 6 } & 3 & 1.7 & 0.2 & 0.5 & 170 \\
\cline { 2 - 6 } & 4 & 1.7 & 0.3 & 0 & 170 \\
\cline { 2 - 6 } & 5 & 1.7 & 0 & 0 & 170 \\
\cline { 2 - 6 } & 6 & 1.7 & 1.0 & 0 & 170 \\
\hline
\end{tabular}

The advantages of full profiles can be seen, particularly for locations where the thermal conductivity is observed to rapidly change. For example, in Figure 4 at approximately $6 \mathrm{~m}$ depth, an organic rich layer is observed, and a low thermal conductivity is predicted. This low value has not been identified by discrete tests, and could play an important part in restricting heat transfer. 

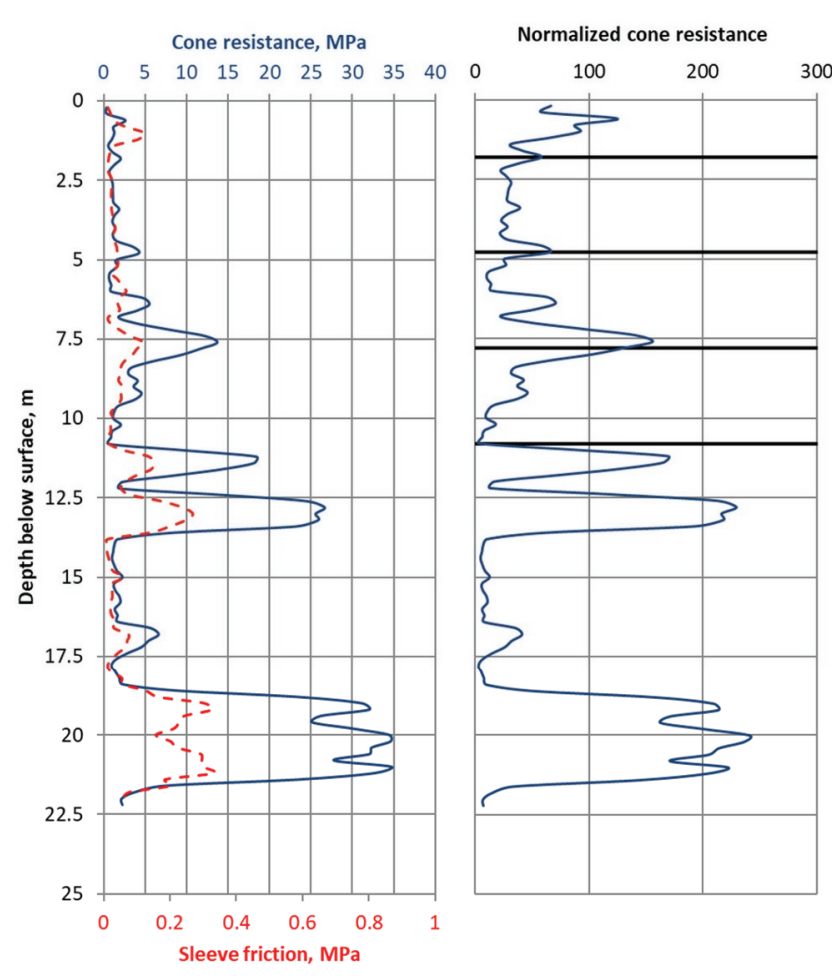

Volumetric heat capacity, $300 \quad 0 \quad 1^{\mathrm{MJ} / \mathrm{m}^{3} \mathrm{~K}} \quad 3 \quad 4$

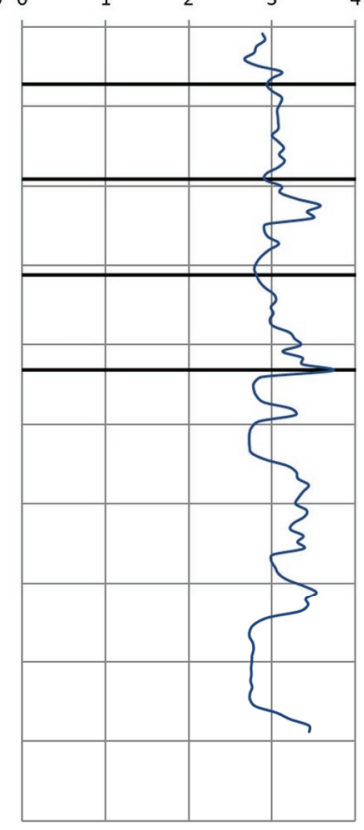

Fig. 4. Ostend site: CPT data, the volumetric heat capacity profile, and the uncorrected and corrected thermal conductivity profiles including discrete data points.
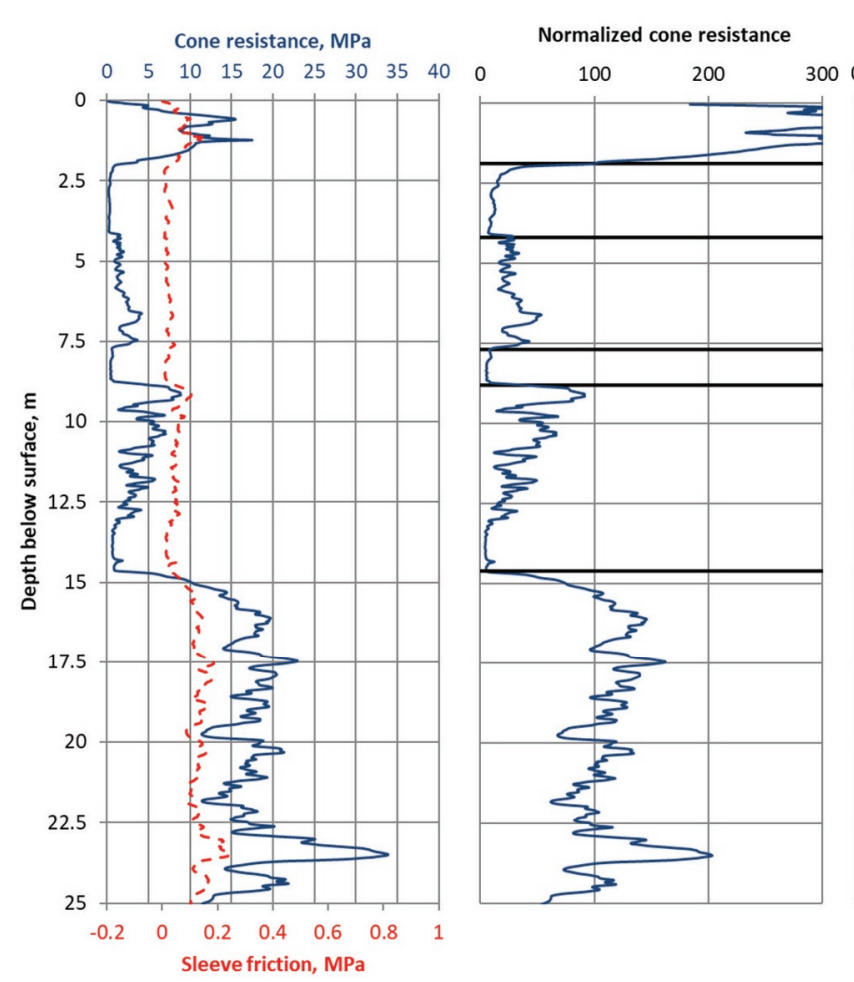
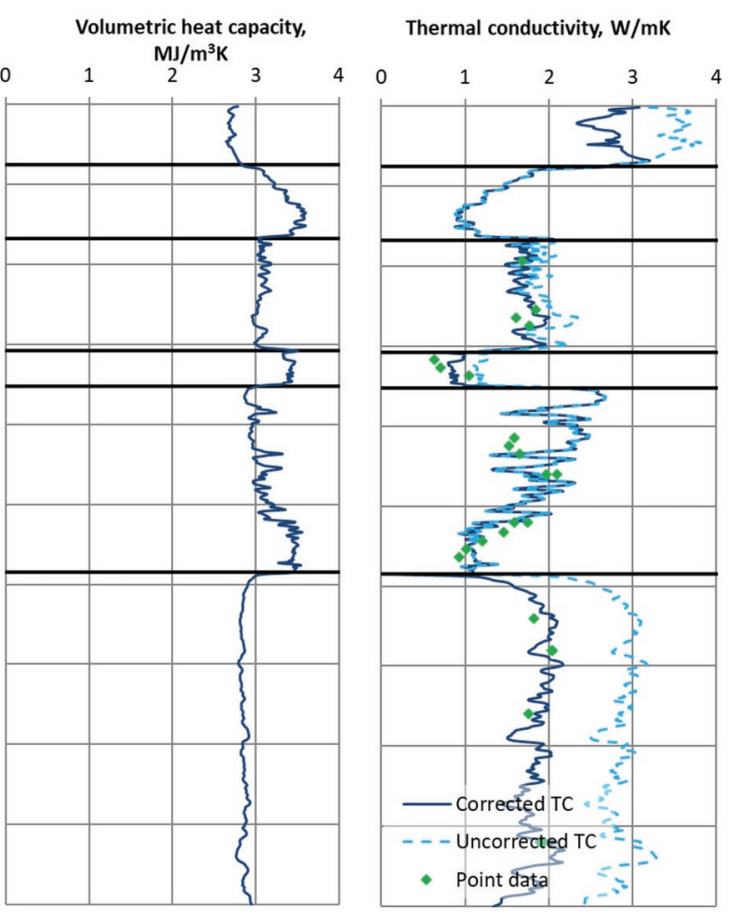

Fig. 5. Delft site: CPT data, the volumetric heat capacity profile, and the uncorrected and corrected thermal conductivity profiles including discrete data points. 


\section{Conclusions}

A method combining two recently proposed CPT based methods of determining profiles of thermal properties of the ground has been demonstrated. The method utilizes both CPT correlations and heat dissipation tests, combining the results together to give accurate and precise estimates of both thermal conductivity and volumetric heat capacity. The data required can be collected at the same time as a CPT-based site investigation, making this method cost-effective and fast for use when designing and installing energygeostructures.

The provision of data by Fugro and the Belgian Building Research Institute (BBRI) is gratefully acknowledged. The data obtained from BBRI were acquired in the framework of Smart Geotherm, a project funded by Flanders Innovation \& Entrepreneurship.

\section{References}

1. P.J. Vardon, D. Baltoukas, J. Peuchen, Interpreting and validating the Thermal Cone Penetration Test (T-CPT). Géotechnique 69, 7 (2019)

2. J.E. Low, F.A. Loveridge, W. Powrie, D. Nicholson, A comparison of laboratory and in situ methods to determine soil thermal conductivity for energy foundations and other ground heat exchanger applications. Acta Geotechnica 10, 2 (2015)

3. ASTM D5334 - 14, Standard test method for determination of thermal conductivity of soil and soft rock by thermal needle probe procedure. (2014)

4. H.S. Carslaw, J.C. Jaeger, Conduction of heat in solids, 2nd edn. (Oxford University Press, London, 1959)

5. ISO 22007-2:2015, Plastics -- Determination of thermal conductivity and thermal diffusivity -- Part 2: Transient plane heat source (hot disc) method. (2015)

6. ISO 17628:2015, Geotechnical investigation and testing - Geothermal testing - Determination of thermal conductivity of soil and rock using a borehole heat exchanger. (2015)

7. VDI 4640 Part 5:2016-08, Thermal use of Underground - Thermal response test. (2016)

8. A. Vieira, J. Maranha, M. Alberdi-Pagola, P. Christodoulides, S. Javed, F. Loveridge, F. Nguyen, F. Cecinato, F. Maranha, G. Florides, I. Prodan, G. van Lysebetten, E. Ramalho, D. Salciarini, A. Georgiev, S Rosin-Paumier, R. Popov, S. Lenart, S. Erbs Poulsen, G. Radioti, Characterisation of ground thermal and thermo-mechanical behaviour for shallow geothermal energy applications. Energies MDPI 10, 12 (2017).

9. G.A. Akrouch, J.L. Briaud, M. Sanchez, R. Yilmaz, Thermal cone test to determine soil thermal properties. J. Geotech. Geoenviron. Engng 142, 3 (2016)
10. J.C. Jaeger, Conduction of heat in an infinite region bounded internally by a circular cylinder of a perfect conductor. Aust. J. Phys. 9, 2 (1956)

11. ISO 22476-1:2012, Geotechnical investigation and testing. Field testing. Electrical cone and piezocone penetration test. (2012)

12. P.J. Vardon, J. Peuchen, CPT correlations for thermal properties of soils. Under review. 